\title{
Fast Random Walk Graph Kernel
}

\author{
U Kang \\ Carnegie Mellon University
}

\author{
Hanghang Tong \\ IBM T.J. Watson
}

\author{
Jimeng Sun \\ IBM T.J. Watson
}

\begin{abstract}
Random walk graph kernel has been used as an important tool for various data mining tasks including classification and similarity computation. Despite its usefulness, however, it suffers from the expensive computational cost which is at least $O\left(n^{3}\right)$ or $O\left(m^{2}\right)$ for graphs with $n$ nodes and $m$ edges.

In this paper, we propose ARK, a set of fast algorithms for random walk graph kernel computation. ARK is based on the observation that real graphs have much lower intrinsic ranks, compared with the orders of the graphs. ARK exploits the low rank structure to quickly compute random walk graph kernels in $O\left(n^{2}\right)$ or $O(m)$ time.

Experimental results show that our method is up to $97,865 \times$ faster than the existing algorithms, while providing more than $91.3 \%$ of the accuracies.
\end{abstract}

\section{Introduction}

Many real-world, complex objects with structural properties can be naturally modeled as graphs. For instance, the Web can be naturally represented as a graph with the Web pages as nodes and hyper links as edges. In the medical domain, the symptom-lab test graph of a given patient, which can be constructed from his/her medical records, provides a good indicator of the structure information of possible disease s/he carries (e.g., the association between a particular symptom and some lab test, the co-occurrence of different symptom). How can we characterize the difference of the current web graph from the one from the last year to spot potential abnormal activities? How can we measure the similarities among different patients so that we can segment them into different categories?

Graph kernel [43] provides a natural tool to answer the above questions. Among others, one of the most powerful graph kernel is based on random walks, and has been successfully applied to many real world applications (see Section 6 for a review). Despite its success, one main challenge remains open in terms of the scalability. To date, the best known algorithm to compute random walk based graph kernel is cubic in terms of the number of the nodes in the graph. Consequently, most, if not all, of the current random walk graph kernel algorithms quickly become computationally infeasible for the graphs with more than hundreds of nodes.

To address this issue, we propose a family of fast algorithms to compute random walk graph kernel. The key observation is that many real graphs have much lower intrinsic ranks, compared with the orders of the graphs. The heart of our proposed algorithms is to leverage such low-rank structure as an intermediate step to speed up the computation. Our experimental evaluations on many real graphs show that the new proposed methods (1) are much faster than the existing ones (up to $97,865 \times$ speed-up); (2) scale to the graphs with 325,729 nodes that all the existing methods fail to compute; and (3) bear a high approximation accuracy (more than $91.3 \%$ ).

The main contributions of this paper are as follows.

1. Algorithms. We propose ARK, a set of fast algorithms to compute random walk graph kernel, which significantly reduce the time complexity (see Table 2 for a comparison).

2. Proofs and Analysis. We show the approximation error bounds as well as the efficiency of our methods.

3. Experiments. We perform extensive experiments on many real world graphs, showing the speed-up (maximum $97,865 \times$ ) and the accuracy (more than $91.3 \%$ ) of our proposed method.

The rest of paper is organized as follows. Section 2 presents the preliminaries of the standard random walk graph kernel. Sections 3 and 4 describe our proposed ARK algorithms for unlabeled and labeled graphs, respectively. Section 5 presents the experimental results. After reviewing related works in Section 6, we conclude in Section 7.

\section{Preliminaries; Random Walk Graph Kernel}

In this section, we describe the preliminaries on the random walk graph kernel whose fast algorithms will be proposed in Sections 3 and 4. Table 1 lists the symbols used in this paper. 


\begin{tabular}{|c|l|}
\hline Symbol & Definition \\
\hline \hline$G$ & a graph \\
\hline$n$ & number of nodes in a graph \\
\hline$m$ & number of edges in a graph \\
\hline$A$ & adjacency matrix of a graph \\
\hline$k\left(G_{1}, G_{2}\right)$ & $\begin{array}{l}\text { exact graph kernel function on graphs } \\
G_{1} \text { and } G_{2}\end{array}$ \\
\hline$\hat{k}\left(G_{1}, G_{2}\right)$ & $\begin{array}{l}\text { approximate graph kernel function on } \\
\text { graphs } G_{1} \text { and } G_{2}\end{array}$ \\
\hline$W$ & weight matrix in random walk kernel \\
\hline$c$ & decay factor in random walk kernel \\
\hline$d_{n}$ & number of distinct node labels \\
\hline$r$ & $\begin{array}{l}\text { reduced rank after low rank } \\
\text { approximation }\end{array}$ \\
\hline$|\cdot|_{F}$ & Frobenius norm \\
\hline
\end{tabular}

Table 1: Table of symbols.

2.1 Definition Random walk graph kernel has been used for classification and measuring similarities of graphs [20, 43]. Given two graphs, the random walk graph kernel computes the number of common walks in two graphs. Two walks are common if the lengths of the walks are equal, and the label sequences are the same (for nodes/edges labeled graphs). The computed number of common walks is used to measure the similarity of two graphs.

We derive the random walk graph kernel for the unlabeled and unnormalized cases, and generalize the definition to labeled and normalized cases. Given two graphs $G_{1}=\left\{V_{1}, E_{1}\right\}$ and $G_{2}=\left\{V_{2}, E_{2}\right\}$, the direct product graph $G_{\times}=\left\{V_{\times}, E_{\times}\right\}$of $G_{1}$ and $G_{2}$ is a graph with the node set $V_{\times}=\left\{\left(v_{1}, v_{2}\right) \mid v_{1} \in V_{1}, v_{2} \in V_{2}\right\}$, and the edge set $E_{\times}=\left\{\left(\left(v_{11}, v_{21}\right),\left(v_{12}, v_{22}\right)\right) \mid\left(v_{11}, v_{12}\right) \in\right.$ $\left.E_{1},\left(v_{21}, v_{22}\right) \in E_{2}\right\}$. A random walk on the direct product graph $G_{\times}$is equivalent to the simultaneous random walks on $G_{1}$ and $G_{2}$. Let $p_{1}$ and $p_{2}$ be the starting probabilities of the random walks on $G_{1}$ and $G_{2}$, respectively. The stopping probabilities $q_{1}$ and $q_{2}$ are defined similarly. Then, the number of length $l$ common walks on the direct product graph $G_{\times}$is given by $\left(q_{1} \otimes q_{2}\right)\left(W_{1}^{T} \otimes W_{2}^{T}\right)^{l}\left(p_{1} \otimes p_{2}\right)$, where $W_{1}$ and $W_{2}$ are the adjacency matrices of $G_{1}$ and $G_{2}$, respectively [43]. Discounting the longer walks by the decay factor $c$, and summing up all the common walks for all different lengths, we derive the equation for the random walk graph kernel:

$$
\begin{aligned}
k\left(G_{1}, G_{2}\right) & =\quad \sum_{l=0}^{\infty}\left(q_{1} \otimes q_{2}\right)\left(W_{1}^{T} \otimes W_{2}^{T}\right)^{l}\left(p_{1} \otimes p_{2}\right) \\
& =\left(q_{1} \otimes q_{2}\right)\left(I-c\left(W_{1}^{T} \otimes W_{2}^{T}\right)\right)^{-1}\left(p_{1} \otimes p_{2}\right) .
\end{aligned}
$$

More generally, the random walk graph kernel is defined as follows.

\section{Definition 1. (RAndom Walk Graph Kernel)}

Let $G_{1}$ and $G_{2}$ be two graphs. Let $p_{1}$ and $p_{2}$ be the starting probabilities of the random walks on $G_{1}$ and $G_{2}$, respectively. Similarly, let $q_{1}$ and $q_{2}$ be the stopping probabilities of the random walks on $G_{1}$ and $G_{2}$, respectively. The random walk graph kernel $k\left(G_{1}, G_{2}\right)$ is determined by

$$
k\left(G_{1}, G_{2}\right):=q^{T}(I-c W)^{-1} p,
$$

where $W$ is a weight matrix, $c$ is a decay factor, $p=p_{1} \otimes p_{2}$, and $q=q_{1} \otimes q_{2}$.

The weight matrix $W$ is determined by two factors: normalization and labels on nodes/edges.

Normalization. Let $A_{1}$ and $A_{2}$ be the adjacency matrices of $G_{1}$ and $G_{2}$, respectively. For unnormalized case, the weight matrix is given by $W=A_{1}^{T} \otimes A_{2}^{T}$. For normalized case, the weight matrix is given by $W=A_{1}^{T} D_{1}^{-1} \otimes A_{2}^{T} D_{2}^{-1}$, where $D_{1}$ and $D_{2}$ are diagonal matrices whose $i$ th diagonal elements are given by $\sum_{j} A_{1}(i, j)$ and $\sum_{j} A_{2}(i, j)$, respectively.

Labels. Nodes and edges can be labeled. First, we consider node labeled graphs. Let $G_{1}$ have $n_{1}$ nodes and $G_{2}$ have $n_{2}$ nodes. Let $l_{1}$ and $l_{2}$ be the node label vectors of $G_{1}$ and $G_{2}$, respectively. The $\left((i-1) \cdot n_{2}+j\right)$ th row of the weight matrix $W$ is zeroed out if the $i$ th element $l_{1}(i)$ of $l_{1}$ and the $j$ th element $l_{2}(j)$ of $l_{2}$ do not have the same labels. Note that for this case the starting probability $p=p_{1} \otimes p_{2}$ is also changed similarly: $\left((i-1) \cdot n_{2}+j\right)$ th element of $p$ is zeroed out if the $i$ th element $l_{1}(i)$ of $l_{1}$ and the $j$ th element $l_{2}(j)$ of $l_{2}$ do not have the same labels. Second, we consider edge labeled graphs. Let $W_{1}$ and $W_{2}$ be the normalized or unnormalized adjacency matrices of $G_{1}$ and $G_{2}$, respectively. The $\left(\left(i_{1}-1\right) \cdot n_{2}+i_{2},\left(j_{1}-1\right) \cdot n_{2}+j_{2}\right)$ th element of $W$ is 1 if and only if the edge labels of $W_{1}^{T}\left(i_{1}, j_{1}\right)$ and $W_{2}^{T}\left(i_{2}, j_{2}\right)$ are the same.

\subsection{Computing Random Walk Graph Kernel}

We describe methods for computing the random walk graph kernel. For simplicity, we assume that both the graphs $G_{1}$ and $G_{2}$ have $n$ nodes and $m$ edges.

Naive Method. The naive algorithm is to compute the Equation (2.1) by inverting the $n^{2} \times n^{2}$ matrix 
$W$. Since inverting a matrix takes time proportional to the cube of the number of rows/columns, the running time is $O\left(n^{6}\right)$.

Sylvester Method. If the weight matrix can be decomposed into one or two sums of Kronecker products, Sylvester method solves the Equation (2.1) in $O\left(n^{3}\right)$ time [43]. However, there are two drawbacks in Sylvester method. First, the method requires the two graphs to have the same number of nodes, which is often not true. Second, the theoretical running time of Sylvester method on the weight matrix composed of more than two Kronecker products is unknown.

Spectral Decomposition Method. For unlabeled and unnormalized matrices, spectral decomposition method runs in $O\left(n^{3}\right)$ time. The problem of spectral decomposition method is that it can't run on the labeled graph or normalized matrix.

Conjugate Gradient Method. Conjugate gradient (CG) method is used to solve linear systems efficiently. To use CG for computing random walk graph kernel, we first solve $(I-c W) x=p$ for $x$ using $\mathrm{CG}$, and compute $q^{T} x$. Each iteration of CG takes $O\left(m^{2}\right)$ since the most expensive operation is the matrix-vector multiplication. Thus CG takes $O\left(m^{2} i_{F}\right)$ time where $i_{F}$ denote the number of iterations. A problem of the CG method is its high memory requirement: it requires $O\left(m^{2}\right)$ memory.

Fixed Point Iteration Method. Fixed point iteration method first solves $(I-c W) x=p$ for $x$ by iterative matrix-vector multiplications, and then computes $q^{T} x$ to compute the kernel. Note that the fixed point iteration method converges only when the decay factor $c$ is smaller than $\left|\xi_{1}\right|^{-1}$ where $\xi_{1}$ is the largest magnitude eigenvalue of $W$. Similar to CG, the fixed point iteration method takes $O\left(m^{2} i_{F}\right)$ time for $i_{F}$ iterations, and has the same problems of requiring $O\left(m^{2}\right)$ memory.

Since the Sylvester method and the spectral decomposition method cannot be used for kernels on general graphs, we use the conjugate gradient and the fixed point iterations method for experimental evaluations in Section 5 .

\section{Proposed Approximation: Unlabeled Graphs}

As we saw in Section 2, the exact algorithms to compute the random walk graph kernel take too much time or require too much memory. To solve the problem, we propose ARK (Approximate Random walk Kernel), a set of approximation algorithms to compute the random walk graph kernel in this and the next sections. Table 2 shows the summary of the running time comparison of our ARK and the exact algorithms. This section addresses unlabeled graphs which correspond to the cases (a) and (b) in Table 2. The node labeled graphs, which correspond to the cases (c) and (d) in Table 2, are handled in the next section.

3.1 Asymmetric W (Ark-U) We first consider node unlabeled graphs with the normalized weight matrix, which correspond to the case (a) in Table 2. Let two graphs $G_{1}$ and $G_{2}$ have the adjacency matrices $A_{1}$ and $A_{2}$, respectively. Let $W_{1}=A_{1} D_{1}^{-1}$ and $W_{2}=A_{2} D_{2}^{-1}$ be the row normalized adjacency matrix of $G_{1}$ and $G_{2}$, where $D_{1}$ and $D_{2}$ are diagonal matrices whose $i$ th diagonal elements are given by $\sum_{j} A_{1}(i, j)$ and $\sum_{j} A_{2}(i, j)$, respectively. In this setting, the weight matrix $W$ is given by

$$
W=W_{1}^{T} \otimes W_{2}^{T} .
$$

Since the $W$ matrix is large, we approximate $W$ using its low-rank approximation. More precisely, we define the $r$-approximation of a matrix as follows.

Definition 2. ( $r$-Approximation of A MATRix)

Given a matrix $A$, the r-approximation $\hat{A}$ of $A$ is a matrix satisfying the following equation:

$$
\hat{A}=\sum_{\sigma_{i} \in S_{A}} \sigma_{i} u_{i} u_{i}^{T},
$$

where $u_{i}$ is a singular vector of $A$ with $\sigma_{i}$ as the corresponding singular value, and $S_{A}$, a subset of the set of singular values of $A$, contains top $r$ singular values of $A$.

The $r$-approximation gives a good approximation of the original matrix since the singular value decomposition (SVD) gives the best low rank approximation [41].

Our goal is an approximate random walk kernel which is defined as follows.

\section{Definition 3. (ApProx. RAndom Walk Kernel)}

Given a random walk graph kernel function $k\left(G_{1}, G_{2}\right):=q^{T}(I-c W)^{-1} p$, the approximate random walk graph kernel $\hat{k}\left(G_{1}, G_{2}\right)$ is given by

$$
\hat{k}\left(G_{1}, G_{2}\right):=q^{T}(I-c \hat{W})^{-1} p,
$$

where $\hat{W}$ is a low rank approximation of $W$.

We want the $\hat{W}$ matrix to be as close as possible to $W$, while preserving a low rank. Ideally, $\hat{W}$ can be a $r$-approximation of $W$. A simple but naive approach to get the $r$-approximation of $W$ is to use rank- $r$ SVD of $W$. However, such method is inefficient since the running time is $O\left(m^{2} r\right)$, and the $W$ matrix needs to be explicitly constructed. Our proposed idea is to use 


\begin{tabular}{|c|c|c|c|c|}
\hline Case & Normalization of W & \# of Node Labels & Exact & Ark \\
\hline (a) & Normalized & 1 & $O\left(n^{3}\right)$ & $O\left(n^{2} r^{4}+r^{6}+m r\right)$ \\
\hline \hline (b) & Unnormalized & 1 & $O\left(n^{3}\right)$ & $O\left((m+n) r+r^{2}\right)$ \\
\hline \hline (c) & Normalized & $d_{n}$ & $O\left(m^{2} i_{F}\right)$ & $O\left(d_{n} n^{2} r^{4}+r^{6}+m r\right)$ \\
\hline (d) & Unnormalized & $d_{n}$ & $O\left(m^{2} i_{F}\right)$ & $O\left(d_{n} n^{2} r^{4}+r^{6}+m r\right)$ \\
\hline
\end{tabular}

Table 2: Summary of the running time comparison of our ARK and the exact algorithms. $n$ is the number of nodes, $m$ is the number of edges, $r$ is the reduced rank after low rank approximation, $d_{n}$ is the number of node labels, and $i_{F}$ is the number of iterations in the conjugate gradient or the fixed point iteration methods. Note our proposed ARK is faster than the exact method, since $n>>r$. ARK-U handles the case (a) in Section 3.1. ARK-U+ addresses the case (b) in Section 3.2, and ARK-L deals with the cases (c) and (d) in Section 4.

the top $r$ SVD of $W_{1}^{T}$ and $W_{2}^{T}$ to compute the $r$ approximation of the weight matrix $W$. This approach has another advantage of not needing to explicitly construct the $W$ matrix. We first give the algorithm, called ARK-U, and prove its correctness. Algorithm 1 shows our approximation algorithm.

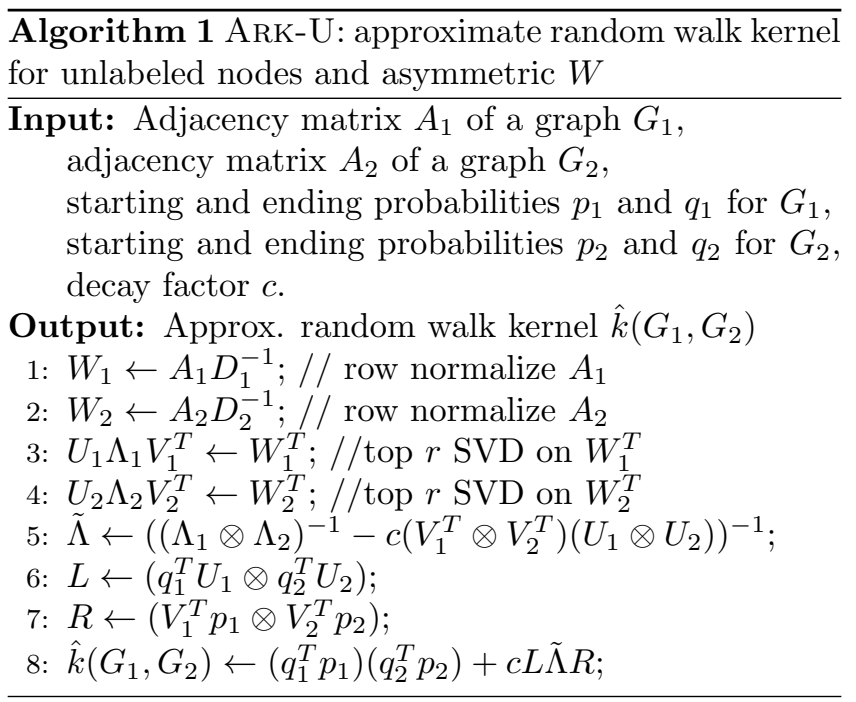

We show that Algorithm 1 is correct.

Theorem 3.1. (Correctness of Ark-U) The Algorithm 1 (ARK-U) gives the approximate random walk kernel

$$
\hat{k}\left(G_{1}, G_{2}\right)=q^{T}(I-c \hat{W})^{-1} p,
$$

where $\hat{W}$ is a r-approximation of $W=W_{1}^{T} \otimes W_{2}^{T}$.

Proof. Let $U_{1} \Lambda_{1} V_{1}^{T}$ and $U_{2} \Lambda_{2} V_{2}^{T}$ be the top $r$ singular value decompositions of $W_{1}^{T}$ and $W_{2}^{T}$, respectively. Let us define $\hat{W}$ as follows:

$$
\begin{aligned}
\hat{W} & :=U_{1} \Lambda_{1} V_{1}^{T} \otimes U_{2} \Lambda_{2} V_{2}^{T} \\
& =\left(U_{1} \otimes U_{2}\right)\left(\Lambda_{1} \otimes \Lambda_{2}\right)\left(V_{1}^{T} \otimes V_{2}^{T}\right) .
\end{aligned}
$$

Then, $\hat{W}$ is a $r$-approximation of $W$ since the diagonal elements of the matrix $\Lambda_{1} \otimes \Lambda_{2}$ contain the top $r$ largest singular values of $W_{1}^{T} \otimes W_{2}^{T}$.

Thus,

$$
\begin{aligned}
& q^{T}(I-c \hat{W})^{-1} p \\
& =q^{T}\left(I-c\left(U_{1} \otimes U_{2}\right)\left(\Lambda_{1} \otimes \Lambda_{2}\right)\left(V_{1}^{T} \otimes V_{2}^{T}\right)\right)^{-1} p \\
& =q^{T}\left(I+c\left(U_{1} \otimes U_{2}\right) \tilde{\Lambda}\left(V_{1}^{T} \otimes V_{2}^{T}\right)\right) p \\
& =q^{T} p+c q^{T}\left(U_{1} \otimes U_{2}\right) \tilde{\Lambda}\left(V_{1}^{T} \otimes V_{2}^{T}\right) p \\
& =\left(q_{1}^{T} p_{1}\right)\left(q_{2}^{T} p_{2}\right)+c\left(q_{1}^{T} U_{1} \otimes q_{2}^{T} U_{2}\right) \tilde{\Lambda}\left(V_{1}^{T} p_{1} \otimes V_{2}^{T} p_{2}\right),
\end{aligned}
$$

where the second equality comes from the ShermanMorrison Lemma [29].

We show the time and the space complexities of Algorithm 1. Note that the time complexity $O\left(n^{2} r^{4}+\right.$ $\left.m r+r^{6}\right)$ of ARK-U is smaller than the best exact algorithm's complexity $O\left(n^{3}\right)$, since $r$ is very small (6 is enough, as we see in Section 5) and considered as a constant.

Theorem 3.2. (Time Complexity of ARK-U) ARK-U takes $O\left(n^{2} r^{4}+m r+r^{6}\right)$ time.

Proof. The top $r$ decompositions in lines 3 and 4 cost $O(m r)$. Computing $\tilde{\Lambda}$ in line 5 takes $O\left(n^{2} r^{4}+r^{6}\right)$. Computing line 6, 7 and 8 takes $O\left(n r+r^{4}\right)$.

Theorem 3.3. (Space Complexity of Ark-U) ARK-U requires $O\left(m+n^{2} r^{2}\right)$ space.

Proof. The storage of $W_{1}$ and $W_{2}$ require $O(m)$ space. The top $r$ decompositions in lines 3 and 4 require $O(n r)$. Line 5 to 8 require $O\left(n^{2} r^{2}\right)$ space, thus making the total space complexity $O\left(m+n^{2} r^{2}\right)$.

3.2 Symmetric W (Ark-U+) ARK-U can be used for both the symmetric and the asymmetric weight matrices. For symmetric weight matrix, however, we propose ARK-U+, an even faster approximation algorithm. ArK-U+ handles the case (b) in Table 2. 
We first describe the weight matrix $W$ in this setting. Assume two graphs $G_{1}$ and $G_{2}$ have the symmetric adjacency matrices $A_{1}$ and $A_{2}$, respectively. Then, the weight matrix $W$ is given by

$$
W=A_{1}^{T} \otimes A_{2}^{T},
$$

where $W$ is also symmetric by the nature of Kronecker products [23]. Our proposed idea is to use the eigen decomposition, instead of SVD, to compute the $r$-approximation of $W$. Since the eigen decomposition and the SVD on symmetric matrices are different only up to signs, the eigen decomposition gives the correct $r$-approximation. The computational advantage is that we need to store only one $n \times r$ eigenvector matrix, instead of two $n \times r$ singular vector matrices. Algorithm 2 shows our ARK-U+ algorithm for symmetric $W$.

Algorithm 2 ARK-U+: approximate random walk kernel for unlabeled nodes and symmetric $W$

Input: Adjacency matrix $A_{1}$ of a graph $G_{1}$,

adjacency matrix $A_{2}$ of a graph $G_{2}$

starting and ending probabilities $p_{1}$ and $q_{1}$ for $G_{1}$, starting and ending probabilities $p_{2}$ and $q_{2}$ for $G_{2}$, decay factor $c$.

Output: Approx. random walk kernel $\hat{k}\left(G_{1}, G_{2}\right)$

1: $U_{1} \Lambda_{1} U_{1}^{T} \leftarrow A_{1}^{T} ; / /$ top $r$ eigen decomposition on $W_{1}$

2: $U_{2} \Lambda_{2} U_{2}^{T} \leftarrow A_{2}^{T} ; / /$ top $r$ eigen decomposition on $W_{2}$

3: $\tilde{\Lambda} \leftarrow\left(\left(\Lambda_{1} \otimes \Lambda_{2}\right)^{-1}-c I\right)^{-1}$;

4: $L \leftarrow\left(q_{1}^{T} U_{1} \otimes q_{2}^{T} U_{2}\right)$;

5: $R \leftarrow\left(U_{1}^{T} p_{1} \otimes U_{2}^{T} p_{2}\right)$;

6: $\hat{k}\left(G_{1}, G_{2}\right) \leftarrow\left(q_{1}^{T} p_{1}\right)\left(q_{2}^{T} p_{2}\right)+c L \tilde{\Lambda} R ;$

We show that Algorithm 2 is correct.

Theorem 3.4. (Correctness of Ark-U+) The Algorithm 2 (ARK-U+) gives the approximate random walk kernel

$$
\hat{k}\left(G_{1}, G_{2}\right)=q^{T}(I-c \hat{W})^{-1} p,
$$

where $\hat{W}$ is a r-approximation of $W=A_{1}^{T} \otimes A_{2}^{T}$.

Proof. Let $U_{1} \Lambda_{1} U_{1}^{T}$ and $U_{2} \Lambda_{2} U_{2}^{T}$ be the top $r$ eigen decompositions of $A_{1}$ and $A_{2}$, respectively. Let us define $\hat{W}$ as follows:

$$
\begin{aligned}
\hat{W} & :=U_{1} \Lambda_{1} U_{1}^{T} \otimes U_{2} \Lambda_{2} U_{2}^{T} \\
& =\left(U_{1} \otimes U_{2}\right)\left(\Lambda_{1} \otimes \Lambda_{2}\right)\left(U_{1}^{T} \otimes U_{2}^{T}\right) .
\end{aligned}
$$

Then, $\hat{W}$ is a $r$-approximation of $W$ since the diagonal elements of the matrix $\Lambda_{1} \otimes \Lambda_{2}$ contain the top $r$ largest eigen values of $A_{1}^{T} \otimes A_{2}^{T}$.
Thus,

$$
\begin{aligned}
& q^{T}(I-c \hat{W})^{-1} p \\
& =q^{T}\left(I-c\left(U_{1} \otimes U_{2}\right)\left(\Lambda_{1} \otimes \Lambda_{2}\right)\left(U_{1}^{T} \otimes U_{2}^{T}\right)\right)^{-1} p \\
& =q^{T}\left(I+c\left(U_{1} \otimes U_{2}\right) \tilde{\Lambda}\left(U_{1}^{T} \otimes U_{2}^{T}\right)\right) p \\
& =q^{T} p+c q^{T}\left(U_{1} \otimes U_{2}\right) \tilde{\Lambda}\left(U_{1}^{T} \otimes U_{2}^{T}\right) p \\
& =\left(q_{1}^{T} p_{1}\right)\left(q_{2}^{T} p_{2}\right)+c\left(q_{1}^{T} U_{1} \otimes q_{2}^{T} U_{2}\right) \tilde{\Lambda}\left(U_{1}^{T} p_{1} \otimes U_{2}^{T} p_{2}\right),
\end{aligned}
$$

where the second equality comes from the ShermanMorrison Lemma [29].

We show the time and the space complexities of Algorithm 2. Note that the time and the space complexities of ARK-U+ are smaller than those of ARK-U due to the exploitation of the symmetricity.

Theorem 3.5. (Time complexity of Ark-U+) ARK-U+takes $O\left((m+n) r+r^{2}\right)$ time.

Proof. The top $r$ decompositions in lines 1 and 2 cost $O(m r)$. Computing $\tilde{\Lambda}$ in line 3 takes $O\left(r^{2}\right)$ since $\tilde{\Lambda}$ is a diagonal matrix with $\frac{\lambda_{1}^{i} \lambda_{2}^{j}}{1-c \lambda_{1}^{i} \lambda_{2}^{j}}$, for $1 \leq i, j \leq r$, as its elements. Computing line 4,5 and 6 takes $O\left(n r+r^{2}\right)$.

Theorem 3.6. (Space COMPleXity of Ark-U+) ARK-U requires $O\left(m+n r+r^{2}\right)$ space.

Proof. The storage of $W_{1}$ and $W_{2}$ require $O(m)$ space. The top $r$ decompositions in lines 3 and 4 require $O(n r)$. Line 5 to 8 require $O\left(n r+r^{2}\right)$ space, thus making the total space complexity $O\left(m+n r+r^{2}\right)$.

3.3 Error Bound How close is the approximate random walk kernel $\hat{k}\left(G_{1}, G_{2}\right)$ to the exact kernel $k\left(G_{1}, G_{2}\right)$ ? The analysis for general cases is difficult, but for ARK-U+ which handles symmetric $W$ we have the following error bound.

Theorem 3.7. In ArK-U+, the difference of the exact and the approximate random walk kernel is bounded by

$$
\left|k\left(G_{1}, G_{2}\right)-\hat{k}\left(G_{1}, G_{2}\right)\right| \leq \sum_{(i, j) \notin H}\left|\frac{c \lambda_{1}^{(i)} \lambda_{2}^{(j)}}{1-c \lambda_{1}^{(i)} \lambda_{2}^{(j)}}\right|
$$

where $\lambda_{1}^{(i)}$ and $\lambda_{2}^{(i)}$ are the ith largest eigenvalue of $\Lambda_{1}$ and $\Lambda_{2}$, respectively, and $H=\{(a, b) \mid a, b \in[1, k]\}$ is the set of pairs $(a, b)$ where both $a$ and $b$ are in the range of $[1, k]$.

Proof. Let $W=A_{1}^{T} \otimes A_{2}^{T}$. Then, $\left(U_{1} \otimes U_{2}\right)\left(\Lambda_{1} \otimes\right.$ $\left.\Lambda_{2}\right)\left(U_{1}^{T} \otimes U_{2}^{T}\right)$ is an eigen decomposition of $W$ which 
includes top $k$ largest eigenvalues of $W$. Let $u_{1}^{(i)}$ and $u_{2}^{(i)}$ be the $i$ th column of $U_{1}$ and $U_{2}$, respectively. Then, $\tilde{u}^{(i, j)}:=u_{1}^{(i)} \otimes u_{2}^{(j)}$ is the eigenvector of $W$ with the corresponding eigenvalue $\lambda_{1}^{(i)} \lambda_{2}^{(j)}$. It follows that

$$
\begin{aligned}
& (I-c W)^{-1} \\
& =I+c\left(U_{1} \otimes U_{2}\right) \tilde{\Lambda}\left(U_{1}^{T} \otimes U_{2}^{T}\right) \\
& =I+\sum_{i, j \in[1, n]} \tilde{\lambda}^{(i, j)} \tilde{u}^{(i, j)}\left(\tilde{u}^{(i, j)}\right)^{T},
\end{aligned}
$$

where $\tilde{\Lambda}:=\left(\left(\Lambda_{1} \otimes \Lambda_{2}\right)^{-1}-c I\right)^{-1}$, and $\tilde{\lambda}^{(i, j)}:=\frac{c \lambda_{1}^{(i)} \lambda_{2}^{(j)}}{1-c \lambda_{1}^{(i)} \lambda_{2}^{(j)}}$.

Now, we consider our approximation. Let $\hat{W}$ be the approximation of the $W$ matrix from the top $k$ low rank approximations of $W_{1}$ and $W_{2}$, as shown in Equation (3.2). Then,

$$
(I-c \hat{W})^{-1}=I+\sum_{i, j \in[1, k]} \tilde{\lambda}^{(i, j)} \tilde{u}^{(i, j)}\left(\tilde{u}^{(i, j)}\right)^{T} .
$$

Thus,

$$
\begin{aligned}
& \left|k\left(G_{1}, G_{2}\right)-\hat{k}\left(G_{1}, G_{2}\right)\right| \\
& =\left|q^{T}(I-c W)^{-1} p-q^{T}(I-c \hat{W})^{-1} p\right| \\
& =\left|q^{T}\left(\sum_{(i, j) \notin H} \frac{c \lambda_{1}^{(i)} \lambda_{2}^{(j)}}{1-c \lambda_{1}^{(i)} \lambda_{2}^{(j)}} \tilde{u}^{(i, j)}\left(\tilde{u}^{(i, j)}\right)^{T}\right) p\right| \\
& \leq|| q^{T}\left\|_{2} \cdot\right\| \sum_{(i, j) \notin H} \frac{c \lambda_{1}^{(i)} \lambda_{2}^{(j)}}{1-c \lambda_{1}^{(i)} \lambda_{2}^{(j)}} \tilde{u}^{(i, j)}\left(\tilde{u}^{(i, j)}\right)^{T}\left\|_{F} \cdot\right\| p \|_{2} \\
& \leq \sum_{(i, j) \notin H}\left|\frac{c \lambda_{1}^{(i)} \lambda_{2}^{(j)}}{1-c \lambda_{1}^{(i)} \lambda_{2}^{(j)}}\right|
\end{aligned}
$$

where in the last inequality we used the fact that $\left\|q^{T}\right\|_{2} \leq\left\|q^{T}\right\|_{1}=1$,

$\|p\|_{2} \leq\|p\|_{1}=1$, and

$\left\|\sum_{i} a_{i} u_{i} u_{i}^{T}\right\|_{F}=\sqrt{\operatorname{tr}\left(\sum_{i} a_{i}^{2} u_{i} u_{i}^{T}\right)}=$

$\sqrt{\sum_{i} a_{i}^{2} \cdot \operatorname{tr}\left(u_{i} u_{i}^{T}\right)}=\sqrt{\sum_{i} a_{i}^{2}} \leq \sum_{i}\left|a_{i}\right|$ for any real numbers $a_{i}$ and orthonormal vectors $u_{i}$.

\section{Proposed Approximation: Labeled Graphs}

In this section, we describe ARK-L, an approximation algorithm to compute the random walk graph kernel on node labeled graphs. As discussed in the beginning of Section 3, ARK-L addresses the cases (c) and (d) in Table 2.

4.1 Weight Matrix As we saw in Section 2, the weight matrix $W$ for node labeled graphs is constructed by zeroing out rows of the Kronecker products of normalized or unnormalized matrices. Specifically, given the normalized or unnormalized adjacency matrices $W_{1}$ and $W_{2}$ of $G_{1}$ and $G_{2}$, respectively, the weight matrix $W$ is given by

$$
W=\tilde{L}\left(W_{1}^{T} \otimes W_{2}^{T}\right),
$$

where $\tilde{L}$ is a diagonal matrix whose $(i, i)$ th element is 0 if the $i$ th row of $\left(W_{1}^{T} \otimes W_{2}^{T}\right)$ is zeroed out due to label inconsistency, or 1 otherwise. Let $L_{1}^{(j)}$ be a diagonal label matrix whose $i$ th element is 1 if the node $i$ of the graph $G_{1}$ has the label $j$, and 0 otherwise. $L_{2}^{(j)}$ is defined similarly for the graph $G_{2}$. Then, $\tilde{L}$ is expressed by the sums of Kronecker products:

$$
\tilde{L}=\sum_{j=1}^{d_{n}} L_{1}^{(j)} \otimes L_{2}^{(j)},
$$

where $d_{n}$ is the number of distinct node labels. Finally, the starting probability $p=p_{1} \otimes p_{2}$ is changed to $\tilde{L} p=\tilde{L}\left(p_{1} \otimes p_{2}\right)$ since the random walk is not started for nodes with inconsistent labels.

4.2 Approximation (Ark-L) We first show the approximation algorithm, ARK-L, for random walk kernel on node labeled graphs, and show its correctness. Algorithm 3 shows our approximation algorithm. We assume that $W_{1}$ and $W_{2}$ can be either row-normalized or unnormalized adjacency matrix of $G_{1}$ and $G_{2}$, respectively.

Algorithm 3 ARK-L: approximate random walk kernel for labeled nodes

Input: Weight matrix $W_{1}$ of a graph $G_{1}$,

weight matrix $W_{2}$ of a graph $G_{2}$, label matrices $L_{1}^{(1)}$ to $L_{1}^{\left(d_{n}\right)}$ of $G_{1}$, label matrices $L_{2}^{(1)}$ to $L_{2}^{\left(d_{n}\right)}$ of $G_{2}$, starting and ending probability $p_{1}$ and $q_{1}$ for $G_{1}$, starting and ending probability $p_{2}$ and $q_{2}$ for $G_{2}$, decay factor $c$.

Output: Approx. random walk kernel $\hat{k}\left(G_{1}, G_{2}\right)$

1: $U_{1} \Lambda_{1} V_{1}^{T} \leftarrow W_{1}^{T} ; / /$ top $r$ SVD on $W_{1}^{T}$

2: $U_{2} \Lambda_{2} V_{2}^{T} \leftarrow W_{2}^{T} ; / /$ top $r$ SVD on $W_{2}^{T}$

3: $\tilde{\Lambda} \leftarrow\left(\left(\Lambda_{1} \otimes \Lambda_{2}\right)^{-1}-c\left(\sum_{j=1}^{d_{n}} V_{1}^{T} L_{1}^{(j)} U_{1} \otimes\right.\right.$ $\left.\left.V_{2}^{T} L_{2}^{(j)} U_{2}\right)\right)^{-1}$

4: $L \leftarrow\left(\sum_{j=1}^{d_{n}} q_{1}^{T} L_{1}^{(j)} U_{1} \otimes q_{2}^{T} L_{2}^{(j)} U_{2}\right) ;$

5: $R \leftarrow\left(\sum_{j=1}^{d_{n}} V_{1}^{T} L_{1}^{(j)} p_{1} \otimes V_{2}^{T} L_{2}^{(j)} p_{2}\right)$;

6: $\hat{k}\left(G_{1}, G_{2}\right) \leftarrow\left(\sum_{j=1}^{d_{n}}\left(q_{1}^{T} L_{1}^{(j)} p_{1}\right)\left(q_{2}^{T} L_{2}^{(j)} p_{2}\right)\right)+c L \tilde{\Lambda} R$;

We show that Algorithm 3 is correct. 
Theorem 4.1. (Correctness of Ark-L) The Algorithm 3 (ARK-L) gives the approximate random walk kernel

$$
\hat{k}\left(G_{1}, G_{2}\right)=q^{T}(I-c \hat{W})^{-1} \tilde{L} p,
$$

where $\hat{W}=\tilde{L} \hat{W}_{r}$, and $\hat{W}_{r}$ is a r-approximation of $W_{1}^{T} \otimes W_{2}^{T}$.

Proof. Let $U_{1} \Lambda_{1} V_{1}^{T}$ and $U_{2} \Lambda_{2} V_{2}^{T}$ be the top $r$ singular value decompositions of $W_{1}$ and $W_{2}$, respectively. Let us define $\hat{W}_{r}$ as follows:

$$
\begin{aligned}
\hat{W}_{r} & :=U_{1} \Lambda_{1} V_{1}^{T} \otimes U_{2} \Lambda_{2} V_{2}^{T} \\
& =\left(U_{1} \otimes U_{2}\right)\left(\Lambda_{1} \otimes \Lambda_{2}\right)\left(V_{1}^{T} \otimes V_{2}^{T}\right) .
\end{aligned}
$$

Then, $\hat{W}_{r}$ is a $r$-approximation of $W_{1}^{T} \otimes W_{2}^{T}$ since the diagonal elements of the matrix $\Lambda_{1} \otimes \Lambda_{2}$ contain the top $r$ largest singular values of $W_{1}^{T} \otimes W_{2}^{T}$.

Thus,

$$
\begin{aligned}
& q^{T}\left(I-c \tilde{L} \hat{W}_{r}\right)^{-1} \tilde{L} p \\
& =q^{T}\left(I-c \tilde{L}\left(U_{1} \otimes U_{2}\right)\left(\Lambda_{1} \otimes \Lambda_{2}\right)\left(V_{1}^{T} \otimes V_{2}^{T}\right)\right)^{-1} \tilde{L} p \\
& =q^{T}\left(I+c \tilde{L}\left(U_{1} \otimes U_{2}\right) \tilde{\Lambda}\left(V_{1}^{T} \otimes V_{2}^{T}\right)\right) \tilde{L} p \\
& =q^{T} \tilde{L} p+c q^{T}\left(\sum_{j=1}^{d_{n}} L_{1}^{j} U_{1} \otimes L_{2}^{j} U_{2}\right) \tilde{\Lambda}\left(V_{1}^{T} \otimes V_{2}^{T}\right) \tilde{L} p \\
& =\left(\sum_{j=1}^{d_{n}}\left(q_{1}^{T} L_{1}^{(j)} p_{1}\right)\left(q_{2}^{T} L_{2}^{(j)} p_{2}\right)\right)+c . \\
& \left(\sum_{j=1}^{d_{n}} q_{1}^{T} L_{1}^{(j)} U_{1} \otimes q_{2}^{T} L_{2}^{(j)} U_{2}\right) \tilde{\Lambda}\left(\sum_{j=1}^{d_{n}} V_{1}^{T} L_{1}^{(j)} p_{1} \otimes V_{2}^{T} L_{2}^{(j)} p_{2}\right)
\end{aligned}
$$

where the second equality comes from the ShermanMorrison Lemma [29].

We show the time and the space complexities of Algorithm 3. Note that the time complexity $O\left(d_{n} n^{2} r^{4}+\right.$ $\left.m r+r^{6}\right)$ of ARK-L is smaller than the best exact algorithm's complexity $O\left(m^{2} i_{F}\right)$ since $n>r$ and $n>d_{n}$.

Theorem 4.2. (Time COMPleXity of ARK-L) ARK-L takes $O\left(d_{n} n^{2} r^{4}+m r+r^{6}\right)$ time.

Proof. The top $r$ decompositions in lines 1 and 2 cost $O(m r)$. Computing $\tilde{\Lambda}$ in line 3 takes $O\left(d_{n} n^{2} r^{4}+r^{6}\right)$. Computing line 4, 5 and 6 takes $O\left(d_{n} n r+d_{n} r^{2}+r^{4}\right)$.

Theorem 4.3. (Space Complexity of Ark-L) ARK-L requires $O\left(m+n^{2} r^{2}\right)$ space.
Proof. The storage of $W_{1}$ and $W_{2}$ require $O(m)$ space. The top $r$ decompositions in lines 1 and 2 require $O(n r)$. Line 5 to 8 require $O\left(n^{2} r^{2}\right)$ space, thus making the total space complexity $O\left(m+n^{2} r^{2}\right)$.

\section{Experiments}

We perform experiments to answer the following questions.

Q1 How fast are our ARK algorithms compared to exact methods?

Q2 What are the accuracies of our ARK algorithms compared to exact methods?

Q3 How do the accuracies of ARK change with the number of eigenvalues?

Q1 is answered in Section 5.2, and $\mathrm{Q}(2,3)$ are answered in Section 5.3.

5.1 Experimental Setting For the exact methods, we run both the conjugate gradient and the fixed point iterations, and choose the one with the smaller running time. We use the graphs in Table 3 with the following details.

- WWW-Barabasi: a Web graph snapshot of nd.edu domain.

- HEP-TH: a citation network in the area of theoretical high energy physics.

- AS-Oregon: a router connection graph.

\begin{tabular}{|l|r|r|}
\hline Name & Nodes & Edges \\
\hline \hline WWW-Barabasi & 325,729 & $2,207,671$ \\
\hline HEP-TH & 27,400 & 704,036 \\
\hline AS-Oregon & 13,579 & 74,896 \\
\hline
\end{tabular}

Table 3: Summary of graphs used.

We use the decay factor $c=0.1$ for ARK-U and ARK-L. For ARK-U+, we choose $c=0.0001$ since it was the largest $c$ that allows the fixed point iteration method to converge (see Section 2.2 for more information on the convergence of the fixed point iteration method). All the experiments were performed in a Linux machine with 48 GB memory, and quad-core AMD $2400 \mathrm{MHz}$ CPUs.

5.2 Scalability We first present the scalability results. For each graph, we extract the principal submatrices (=upper, left part of the adjacency matrix) of different lengths, and compute the graph kernel using the two copies of the extracted subgraphs. Figure 1 
shows the running time comparison of our approximation vs. exact methods for real world graphs.

Ark-U. In the first column of Figure 1, ARK-U is compared against the exact method on unlabeled, asymmetric graphs. Note that for all the graphs, ARK$\mathrm{U}$ is $6 \times$ to $11 \times$ faster than the exact method. The exact method is not plotted for all the number of nodes since it failed with the 'out of memory' error.

Ark-U+. In the second column of Figure 1, ARK$\mathrm{U}+$ is compared against the exact method and ARK-U on unlabeled, symmetric graphs. Note that for all the graphs, ARK-U+ is $389 \times$ to $522 \times$ faster than the exact and ARK-U method. The exact and ARK-U method is not plotted for all the number of nodes since they failed with the 'out of memory' error.

Ark-L. Finally, in the third column of Figure 1, ARK-L is compared against the exact method. Note that we omitted the plots for the exact method beyond 500 data points since they took more than 5 hours. Again, ARK-L is $695 \times$ to $97,865 \times$ faster than the exact method.

5.3 Accuracy We present the accuracy of ARK. The accuracy is defined by the relative error of our approximation with regard to the exact kernel:

$$
\text { accuracy }=\frac{\left|\hat{k}\left(G_{1}, G_{2}\right)-k\left(G_{1}, G_{2}\right)\right|}{k\left(G_{1}, G_{2}\right)} .
$$

Accuracy with the Number of Nodes. We fix the number of eigenvalues to 6 , and show the accuracy by increasing the number of nodes. Table 4 shows the result. Note that for all the graphs, ARK gives more than $90 \%$ accuracies. Note also that only top 6 eigenvalues for 2,000 node graph resulted in more than 91.3\% accuracies.

Accuracy with the Number of Eigenvalues. We fix the number of nodes to 500, and show the accuracy by increasing the number of eigenvalues used for the approximation. Table 5 shows the result. Note that for all the graphs, ARK gives more than 90\% accuracies. Note also that increasing the number of eigenvalues increase the accuracy.

\section{Related Work}

The related works branch into three: graph kernel/similarity, node similarity, and low-rank approximation.

Graph Kernel/Similarity. There have been interesting researches in the recent years to classify or measure the similarity between two graphs by kernels. Applications include chemical and biological data classification $[20,32,35]$, and outlier detection [15]. Kernels for discrete objects have been studied by Haussler [14], and many graph kernels have been proposed after that. There are three major groups in graph kernels: kernels based on walks and paths [9, 20, 10, 3, 42, 43], kernels based on limited-size subgraphs [16,36, 21], and kernels based on subtree patterns $[25,35,15]$. Among them, random walk based kernels have been proved to be one of the most successful methods [5, 4, 34]. Vishwanathan et al [43] proposed a unified theoretic framework for various random walk based kernels. However, all the existing algorithms for random walk based kernel require at least cubic running time, which is infeasible for large graphs. There has been works for approximating tree kernels $[33,30,31]$ which are not directly connected to our work. Other remotely related work includes [28], which explored different heuristic to measure the Web graph similarities.

Node Similarity. There are also many works on measuring the similarity between nodes on the same graph, e.g., random walk with restart [27, 39], sinkaugmented delivered current [8], cycle free effective conductance [22], survivable network [13], direction-aware proximity [38], ObjectRank [2], RelationalRank [11], SimRank [18] and its fast approximation [24]

Low-Rank Approximation. Many real world graphs have low intrinsic ranks. Low rank approximation $[12,6,1]$ plays a very important role in mining such graphs, e.g., community detection, anomaly detection, etc. For static graphs, the most popular choices include SVD/PCA $[12,19]$ and random projection [17]. For dynamic graphs, a lot of SVD based techniques have been proposed, such as dynamic tensor analysis [37], incremental spectral clustering [26] etc. More recent work in this line includes example-based low-rank approximation [40], non-negative matrix factorization [44], etc. Finally, there has been works for approximating the SVD itself, using sampling [7].

\section{Conclusions}

In this paper, we propose ARK, fast algorithms for computing random walk kernels. The main contributions are the followings.

1. Algorithms. We carefully design our algorithms to significantly reduce the time complexity.

2. Proofs and Analysis. We give theoretical analysis about the error bound as well as the correctness and the efficiency of our methods.

3. Experiments. We perform numerous experiments on real world graphs, and show that our methods lead to up to $97,865 \times$ speed-up with more than $91.3 \%$ accuracy.

Future research directions include fast random walk kernel computation on time evolving graphs, and de- 

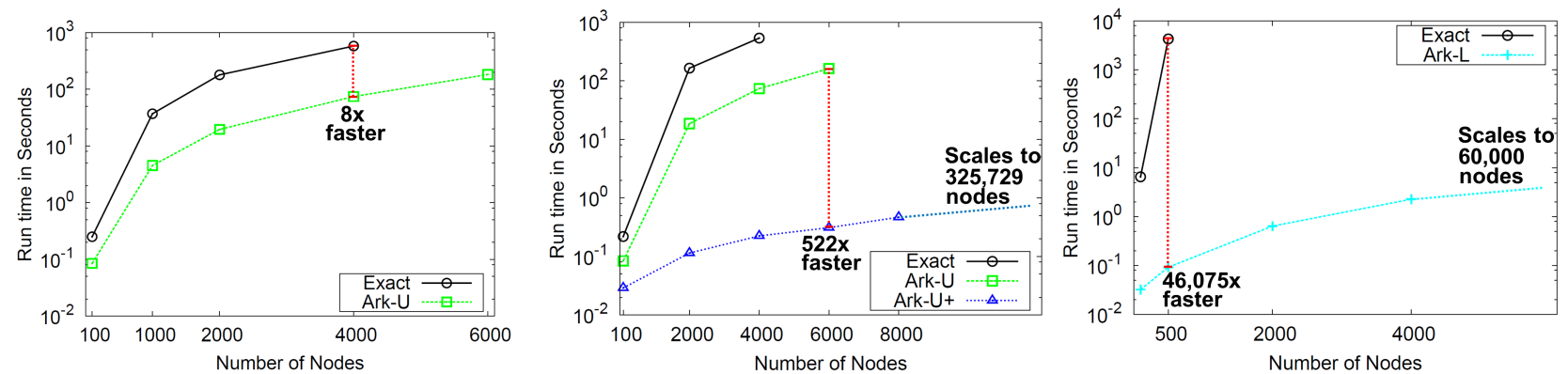

(a) WWW : unlabeled, asymmetric

(b) WWW : unlabeled, symmetric

(c) WWW : labeled
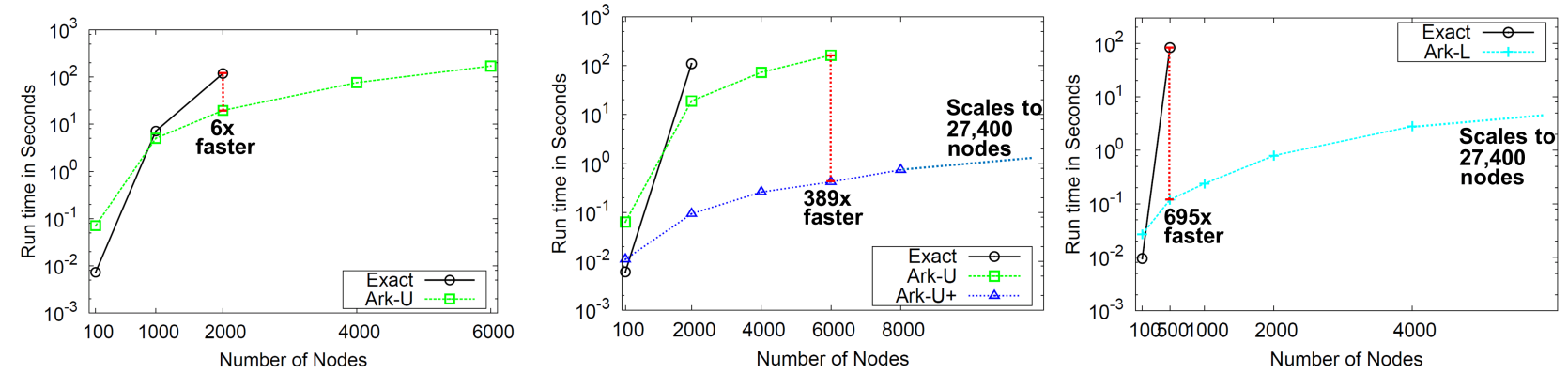

(d) HEP-TH : unlabeled, asymmetric

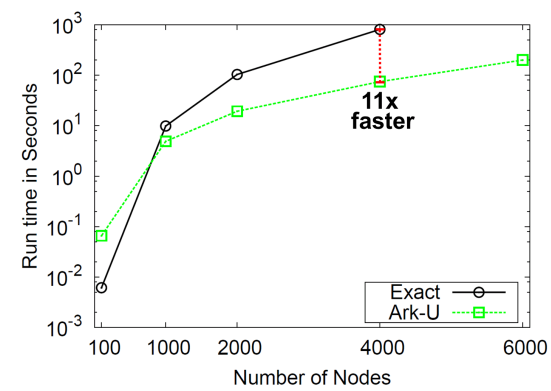

(e) HEP-TH : unlabeled, symmetric

(f) HEP-TH : labeled
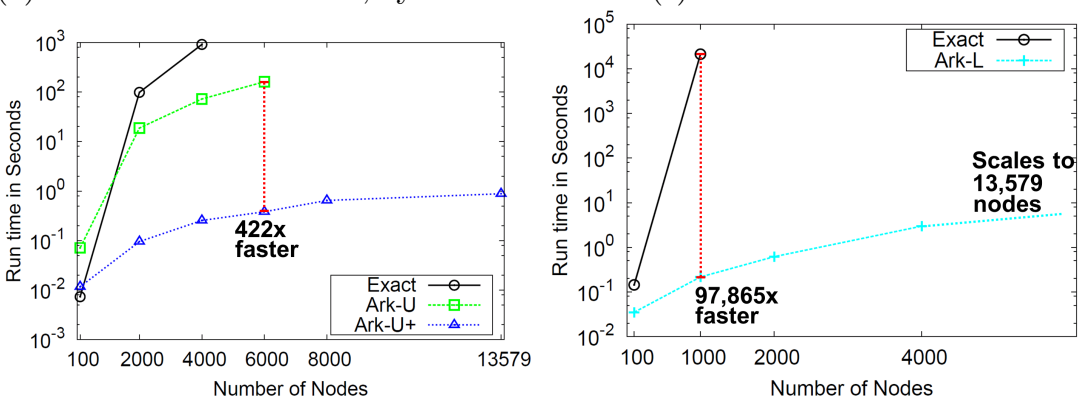

(g) Oregon : unlabeled, asymmetric

(h) Oregon : unlabeled, symmetric

Figure 1: Running time comparison of our approximation vs. exact methods on real world graphs. The Y axes are in $\log$ scale. $(\mathbf{a}, \mathbf{d}, \mathbf{g})$ : ARK-U is $6 \times$ to $11 \times$ faster than the exact method. The exact method is not plotted for all the number of nodes due to 'out of memory' error. (b,e,h): ARK-U+ is $389 \times$ to $522 \times$ faster than the exact and ARK-U method. The exact and the ARK-U method is not plotted fully due to 'out of memory' error. $(\mathbf{c}, \mathbf{f}, \mathbf{i}):$ ARK-L is $695 \times$ to $97,865 \times$ faster than the exact method. We didn't plot the exact method beyond 500 nodes since they took more than 5 hours.

\begin{tabular}{|r|c|c|c|c|c|c|c|c|c|}
\hline & \multicolumn{3}{|c|}{ WWW } & \multicolumn{3}{c|}{ HEP-TH } & \multicolumn{3}{c|}{ Oregon } \\
\hline Nodes & Ark-U & Ark-U+ & Ark-L & Ark-U & Ark-U+ & Ark-L & Ark-U & Ark-U+ & Ark-L \\
\hline \hline 100 & 0.959 & 0.999 & 0.980 & 0.999 & 0.999 & 0.999 & 0.998 & 0.999 & 0.999 \\
\hline 500 & 0.957 & 0.999 & 0.984 & 0.977 & 0.999 & 0.995 & 0.959 & 0.999 & 0.980 \\
\hline 1000 & 0.930 & 0.999 & $*$ & 0.962 & 0.999 & $*$ & 0.939 & 0.999 & $*$ \\
\hline 1500 & 0.920 & 0.999 & $*$ & 0.952 & 0.999 & $*$ & 0.934 & 0.999 & $*$ \\
\hline 2000 & 0.913 & 0.999 & $*$ & 0.946 & 0.998 & $*$ & 0.928 & 0.999 & $*$ \\
\hline
\end{tabular}

Table 4: Accuracy vs. the number of nodes in our approximation. *: could not compute accuracy since the exact method didn't finish. We fixed the number of eigenvalues to 6 . Note the high accuracies for all the graphs, despite the small number of eigenvalues. 


\begin{tabular}{|r|c|c|c|c|c|c|c|c|c|}
\hline & \multicolumn{3}{|c|}{ WWW } & \multicolumn{3}{c|}{ HEP-TH } & \multicolumn{3}{c|}{ Oregon } \\
\hline r* $^{*}$ & Ark-U & Ark-U+ & Ark-L & Ark-U & Ark-U+ & Ark-L & Ark-U & Ark-U+ & Ark-L \\
\hline \hline 1 & 0.930 & 0.993 & 0.971 & 0.977 & 0.999 & 0.996 & 0.943 & 0.999 & 0.970 \\
\hline 2 & 0.930 & 0.999 & 0.971 & 0.977 & 0.999 & 0.996 & 0.947 & 0.999 & 0.974 \\
\hline 3 & 0.950 & 0.999 & 0.981 & 0.977 & 0.999 & 0.996 & 0.950 & 0.999 & 0.975 \\
\hline 4 & 0.951 & 0.999 & 0.981 & 0.977 & 0.999 & 0.996 & 0.952 & 0.999 & 0.976 \\
\hline 5 & 0.953 & 0.999 & 0.982 & 0.977 & 0.999 & 0.995 & 0.954 & 0.999 & 0.977 \\
\hline 6 & 0.957 & 0.999 & 0.984 & 0.977 & 0.999 & 0.995 & 0.959 & 0.999 & 0.980 \\
\hline 7 & 0.961 & 0.999 & 0.986 & 0.977 & 0.999 & 0.995 & 0.961 & 0.999 & 0.981 \\
\hline 8 & 0.962 & 0.999 & 0.987 & 0.977 & 0.999 & 0.995 & 0.961 & 0.999 & 0.981 \\
\hline 9 & 0.962 & 0.999 & 0.987 & 0.977 & 0.999 & 0.995 & 0.964 & 0.999 & 0.983 \\
\hline 10 & 0.963 & 0.999 & 0.987 & 0.977 & 0.999 & 0.994 & 0.966 & 0.999 & 0.984 \\
\hline 11 & 0.965 & 0.999 & 0.989 & 0.977 & 0.999 & 0.994 & 0.967 & 0.999 & 0.984 \\
\hline 12 & 0.966 & 0.999 & 0.989 & 0.977 & 0.999 & 0.994 & 0.968 & 0.999 & 0.985 \\
\hline 13 & 0.967 & 0.999 & 0.989 & 0.977 & 0.999 & 0.994 & 0.969 & 0.999 & 0.986 \\
\hline 14 & 0.969 & 0.999 & 0.991 & 0.977 & 0.999 & 0.994 & 0.970 & 0.999 & 0.986 \\
\hline 15 & 0.971 & 0.999 & 0.991 & 0.977 & 0.999 & 0.994 & 0.971 & 0.999 & 0.986 \\
\hline 16 & 0.971 & 0.999 & 0.991 & 0.977 & 0.999 & 0.994 & 0.972 & 0.999 & 0.987 \\
\hline 17 & 0.972 & 0.999 & 0.992 & 0.977 & 0.999 & 0.993 & 0.974 & 0.999 & 0.988 \\
\hline 18 & 0.972 & 0.999 & 0.992 & 0.977 & 0.999 & 0.993 & 0.976 & 0.999 & 0.989 \\
\hline 19 & 0.972 & 0.999 & 0.992 & 0.977 & 0.999 & 0.993 & 0.977 & 0.999 & 0.989 \\
\hline 20 & 0.973 & 0.999 & 0.992 & 0.977 & 0.999 & 0.993 & 0.977 & 0.999 & 0.990 \\
\hline
\end{tabular}

Table 5: Accuracy vs. the number of eigenvalues used for our approximation on real world graphs. $\mathbf{r}^{*}$ : number of eigenvalues for approximation. Note that first few eigenvalues give more than $90 \%$ of the accuracy.

signing parallel, distributed algorithms for very large graphs which do not fit in the memory of a single machine.

\section{Acknowledgments}

Research was sponsored by the Army Research Laboratory and was accomplished under Cooperative Agreement Number W911NF-09-2-0053. The views and conclusions contained in this document are those of the authors and should not be interpreted as representing the official policies, either expressed or implied, of the Army Research Laboratory or the U.S. Government. The U.S. Government is authorized to reproduce and distribute reprints for Government purposes notwithstanding any copyright notation here on.

\section{References}

[1] D. Achlioptas and F. McSherry. Fast computation of low-rank matrix approximations. J. ACM, 54(2), 2007.

[2] A. Balmin, V. Hristidis, and Y. Papakonstantinou. Objectrank: Authority-based keyword search in databases. In $V L D B$, pages 564-575, 2004.

[3] K. M. Borgwardt and H.-P. Kriegel. Shortest-path kernels on graphs. In ICDM, pages 74-81, 2005.
[4] K. M. Borgwardt, H.-P. Kriegel, S. V. N. Vishwanathan, and N. Schraudolph. Graph kernels for disease outcome prediction from protein-protein interaction networks. In Pacific Symposium on Biocomputing, 2007.

[5] K. M. Borgwardt, S. V. N. Vishwanathan, and H.P. Kriegel. Class prediction from time series gene expression profiles using dynamical systems kernels. In Pacific Symposium on Biocomputing, pages 547-558, 2006.

[6] P. Drineas, R. Kannan, and M. W. Mahoney. Fast monte carlo algorithms for matrices iii: Computing a compressed approximate matrix decomposition. SIAM Journal of Computing, 2005.

[7] P. Drineas, R. Kannan, and M. W. Mahoney. Fast monte carlo algorithms for matrices ii: Computing a low-rank approximation to a matrix. SIAM J. Comput., 36(1):158-183, 2006.

[8] C. Faloutsos, K. S. McCurley, and A. Tomkins. Fast discovery of connection subgraphs. In $K D D$, pages 118-127, 2004.

[9] T. Gärtner, P. A. Flach, and S. Wrobel. On graph kernels: Hardness results and efficient alternatives. In COLT, pages 129-143, 2003.

[10] T. Gärtner, J. W. Lloyd, and P. A. Flach. Kernels and distances for structured data. Machine Learning, 57(3):205-232, 2004. 
[11] F. Geerts, H. Mannila, and E. Terzi. Relational linkbased ranking. In $V L D B$, pages 552-563, 2004.

[12] G. H. Golub and C. F. Van-Loan. Matrix Computations. The Johns Hopkins University Press, Baltimore, 2nd edition, 1996.

[13] M. Grötschel, C. L. Monma, and M. Stoer. Design of survivable networks. In Handbooks in Operations Research and Management Science 7: Network Models. North Holland, 1993.

[14] D. Haussler. Convlution kernels on discrete structures. Technical Report, 1999.

[15] S. Hido and H. Kashima. A linear-time graph kernel. In ICDM, pages 179-188, 2009.

[16] T. Horváth, T. Gärtner, and S. Wrobel. Cyclic pattern kernels for predictive graph mining. In $K D D$, pages 158-167, 2004.

[17] P. Indyk. Stable distributions, pseudorandom generators, embeddings and data stream computation. In FOCS, pages 189-197, 2000.

[18] G. Jeh and J. Widom. Simrank: A measure of structural-context similarity. In $K D D$, pages $538-543$, 2002.

[19] K. V. R. Kanth, D. Agrawal, and A. K. Singh. Dimensionality reduction for similarity searching in dynamic databases. In SIGMOD Conference, pages 166-176, 1998.

[20] H. Kashima, K. Tsuda, and A. Inokuchi. Marginalized kernels between labeled graphs. In ICML, pages $321-$ 328, 2003.

[21] R. I. Kondor, N. Shervashidze, and K. M. Borgwardt. The graphlet spectrum. In ICML, page 67, 2009.

[22] Y. Koren, S. C. North, and C. Volinsky. Measuring and extracting proximity in networks. In $K D D$, pages 245-255, 2006.

[23] A. J. Laub. Matrix analysis for scientists and engineers. SIAM, 2004.

[24] C. Li, J. Han, G. He, X. Jin, Y. Sun, Y. Yu, and T. Wu. Fast computation of simrank for static and dynamic information networks. In EDBT, pages 465-476, 2010.

[25] P. Mahé, N. Ueda, T. Akutsu, J.-L. Perret, and J.-P. Vert. Graph kernels for molecular structureactivity relationship analysis with support vector machines. Journal of Chemical Information and Modeling, 45(4):939-951, 2005.

[26] H. Ning, W. Xu, Y. Chi, Y. Gong, and T. S. Huang. Incremental spectral clustering with application to monitoring of evolving blog communities. In $S D M$, 2007.

[27] J.-Y. Pan, H.-J. Yang, C. Faloutsos, and P. Duygulu. Automatic multimedia cross-modal correlation discovery. In KDD, pages 653-658, 2004.

[28] P. Papadimitriou, A. Dasdan, and H. Garcia-Molina. Web graph similarity for anomaly detection. Journal of Internet Services and Applications, Volume 1(1):1930, May 2010.

[29] W. Piegorsch and G. E. Casella. Inverting a sum of matrices. SIAM Review, 1990.

[30] D. Pighin and A. Moschitti. Reverse engineering of tree kernel feature spaces. In EMNLP, pages 111-120, 2009.

[31] D. Pighin and A. Moschitti. On reverse feature engineering of syntactic tree kernels. In Proceedings of the Fourteenth Conference on Computational Natural Language Learning, pages 223-233, 2010.

[32] L. Ralaivola, S. J. Swamidass, H. Saigo, and P. Baldi. Graph kernels for chemical informatics. Neural Networks, 18(8):1093-1110, 2005.

[33] K. Rieck, T. Krueger, U. Brefeld, and K.-R. Müller. Approximate tree kernels. Journal of Machine Learning Research, 11:555-580, 2010.

[34] H. Saigo, M. Hattori, H. Kashima, and K. Tsuda. Reaction graph kernels predict ec numbers of unknown enzymatic reactions in plant secondary metabolism. BMC Bioinformatics, 11(S-1):31, 2010.

[35] N. Shervashidze and K. M. Borgwardt. Fast subtree kernels on graphs. Advances in Neural Information Processing Systems, 2009.

[36] N. Shervashidze, S. V. N. Vishwanathan, T. Petri, K. Mehlhorn, and K. M. Borgwardt. Efficient graphlet kernels for large graph comparison. Journal of $\mathrm{Ma}$ chine Learning Research - Proceedings Track, 5:488495, 2009.

[37] J. Sun, D. Tao, and C. Faloutsos. Beyond streams and graphs: dynamic tensor analysis. In $K D D$, pages 374 383, 2006.

[38] H. Tong, C. Faloutsos, and Y. Koren. Fast directionaware proximity for graph mining. In $K D D$, pages $747-$ 756, 2007.

[39] H. Tong, C. Faloutsos, and J.-Y. Pan. Random walk with restart: Fast solutions and applications. Knowledge and Information Systems: An International Journal (KAIS), 2008.

[40] H. Tong, S. Papadimitriou, J. Sun, P. S. Yu, and C. Faloutsos. Colibri: fast mining of large static and dynamic graphs. In $K D D$, pages 686-694, 2008.

[41] L. N. Trefethen and D. B. III. Numerical linear algebra. SIAM, 1997.

[42] S. V. N. Vishwanathan, K. M. Borgwardt, and N. N. Schraudolph. Fast computation of graph kernels. In NIPS, pages 1449-1456, 2006.

[43] S. V. N. Vishwanathan, N. N. Schraudolph, R. I. Kondor, and K. M. Borgwardt. Graph kernels. Journal of Machine Learning Research, 11:1201-1242, 2010.

[44] D. Wang, T. Li, and C. H. Q. Ding. Weighted feature subset non-negative matrix factorization and its applications to document understanding. In $I C D M$, pages 541-550, 2010. 